\title{
Enhanced Tumor Penetration and Chemotherapy Efficiency by Covalent Self-assembled Nanomicelle Responsive to Tumor Microenvironment
}

Xiaoqian $\mathrm{Ma}^{\dagger, \ddagger}$, Shuang Bai ${ }^{\dagger, \ddagger}$, Xiaoli Zhang ${ }^{\S}$, Xianbin $\mathrm{Ma}^{\dagger, \ddagger}$, Die $\mathrm{Jia}^{\dagger, \ddagger}$, Xiaoxiao Shi ${ }^{\dagger, \ddagger}$, Jinjun Shao", Peng Xue ${ }^{\dagger, \ddagger}$, Yuejun Kang ${ }^{\dagger, \ddagger}$ and Zhigang $\mathrm{Xu}^{*+, \ddagger}$

${ }^{\dagger}$ Key Laboratory of Luminescent and Real-Time Analytical Chemistry (Southwest University), Ministry of Education, School of Materials and Energy, Southwest University, Chongqing, 400715, P. R. China

${ }^{\ddagger}$ Chongqing Engineering Research Center for Micro-Nano Biomedical Materials and Devices, Chongqing 400715, P. R. China

${ }^{\S}$ Department of Hematology and Oncology, Shenzhen Children's Hospital, Shenzhen, Guangdong 518038, P.R. China

"Key Laboratory of Flexible Electronics (KLOFE) and Institute of Advanced Materials (IAM), Nanjing Tech University (NanjingTech), Nanjing 211816, P.R. China

Email: zgxu@swu.edu.cn or xuzhigang2013@hotmail.com (Z.Xu) 
A

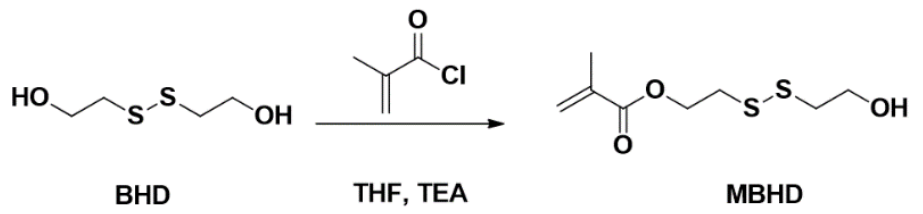<smiles>CCC1(O)C(=O)OCc2c1cc1n(c2=O)Cc2cc3ccccc3nc2-1</smiles>

CPT
(1) $\mathrm{C}_{3} \mathrm{Cl}_{6} \mathrm{O}_{3}$, DMAP, DCM (2) MABHD, DCM

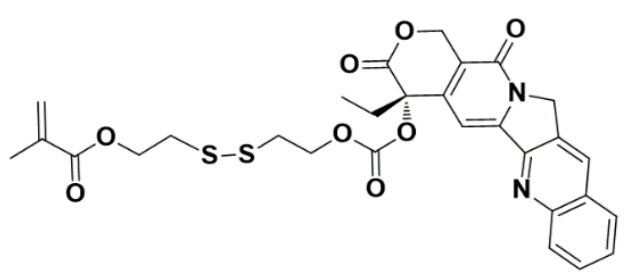

SCPT

B<smiles>C=C(C)C(=O)OCCNC(=O)OC(C)(C)C</smiles>

Figure S1.The synthetic route of MBHD and SCPT monomer (A) and MAEB (B).

A<smiles>CC(=O)C(=O)OCSSCCO</smiles>
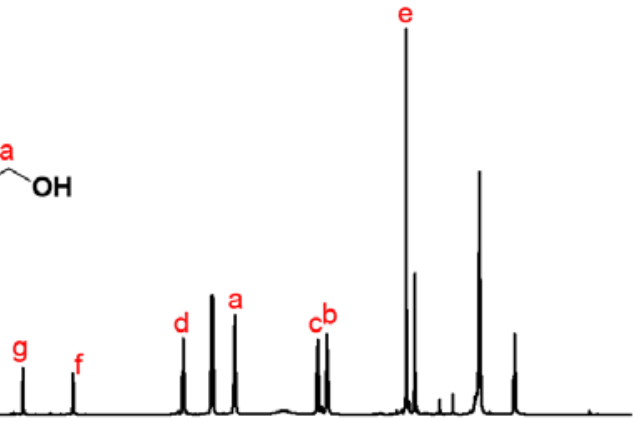

B

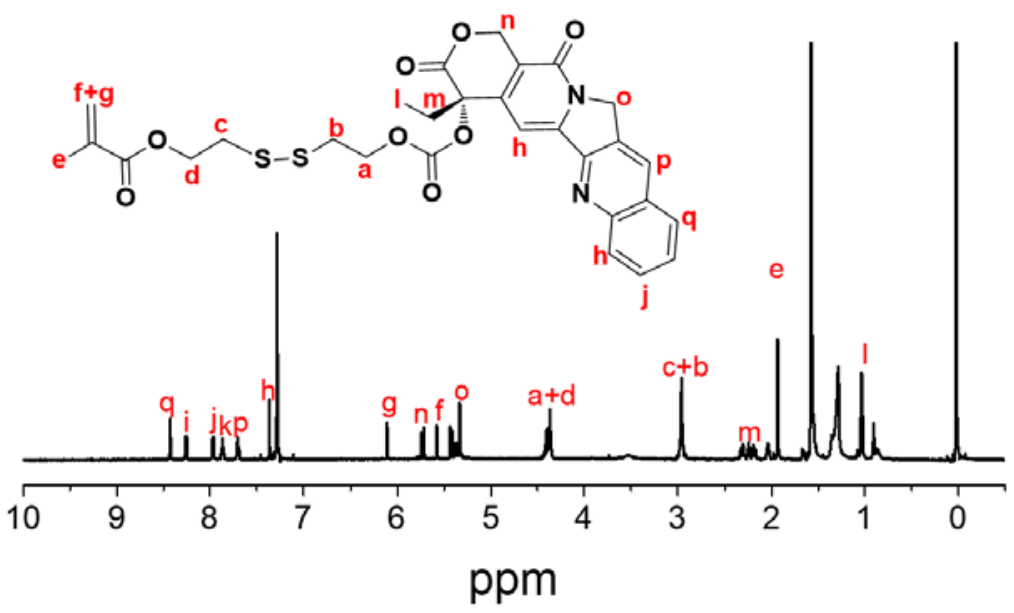

Figure S2. The ${ }^{1} \mathrm{HNMR}$ spectra of MBHD (A) and SCPT (B). 


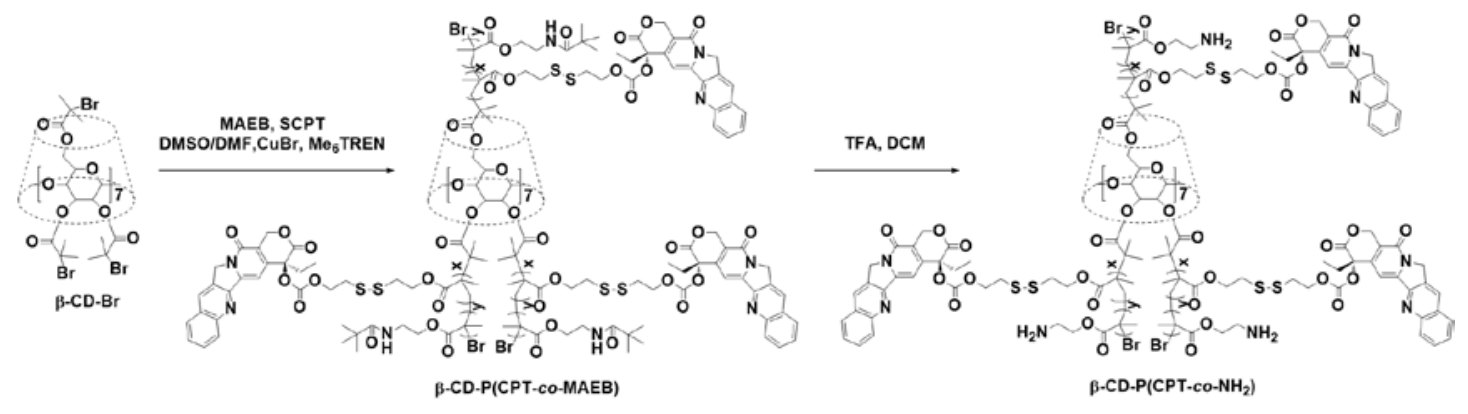

Figure S3. The synthetic route of $\beta-\mathrm{CD}-\mathrm{P}\left(\mathrm{CPT}-\mathrm{co}-\mathrm{NH}_{2}\right)$ (denoted as CD-PCPT).

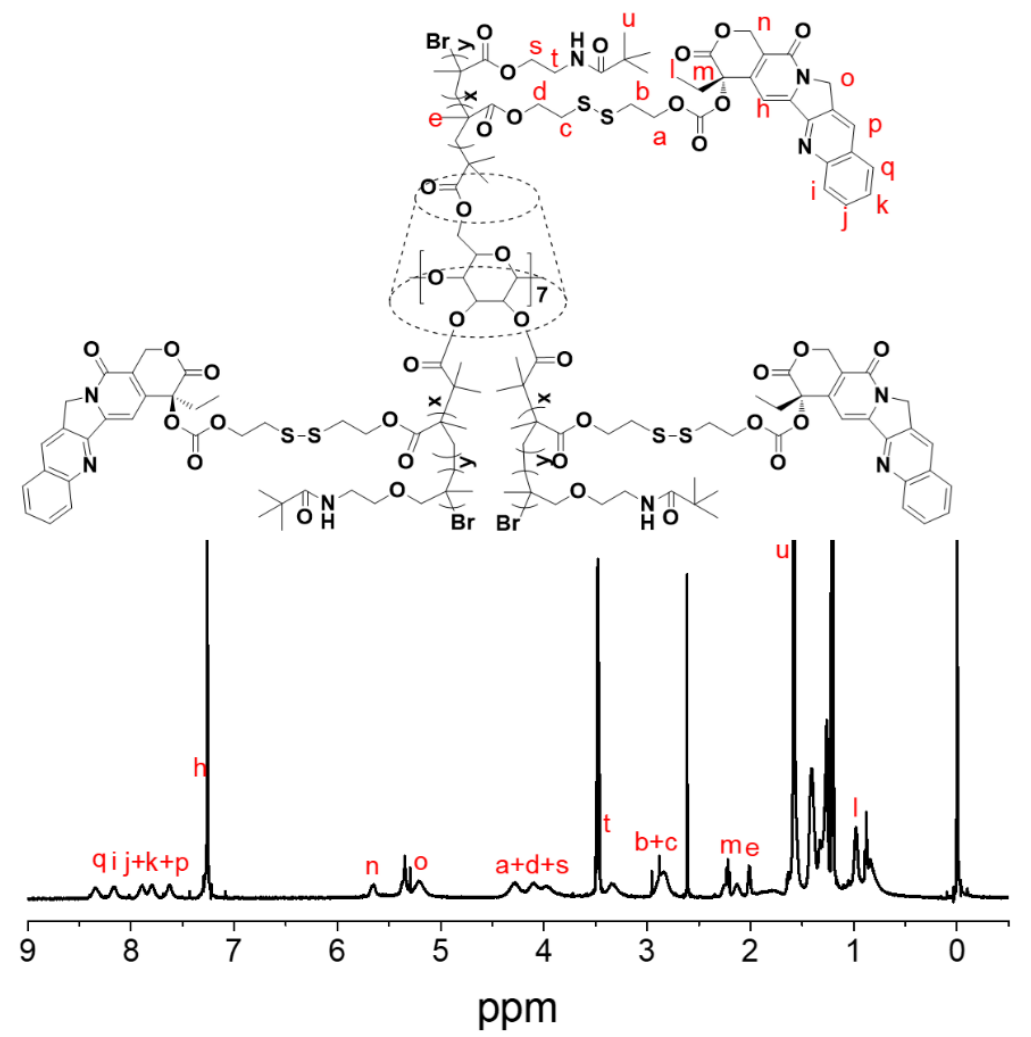

Figure S4. ${ }^{1} \mathrm{HNMR}$ spectrum of $\beta$-CD-P (CPT-co-MAEB) detected in $\mathrm{CDCl}_{3}$. 


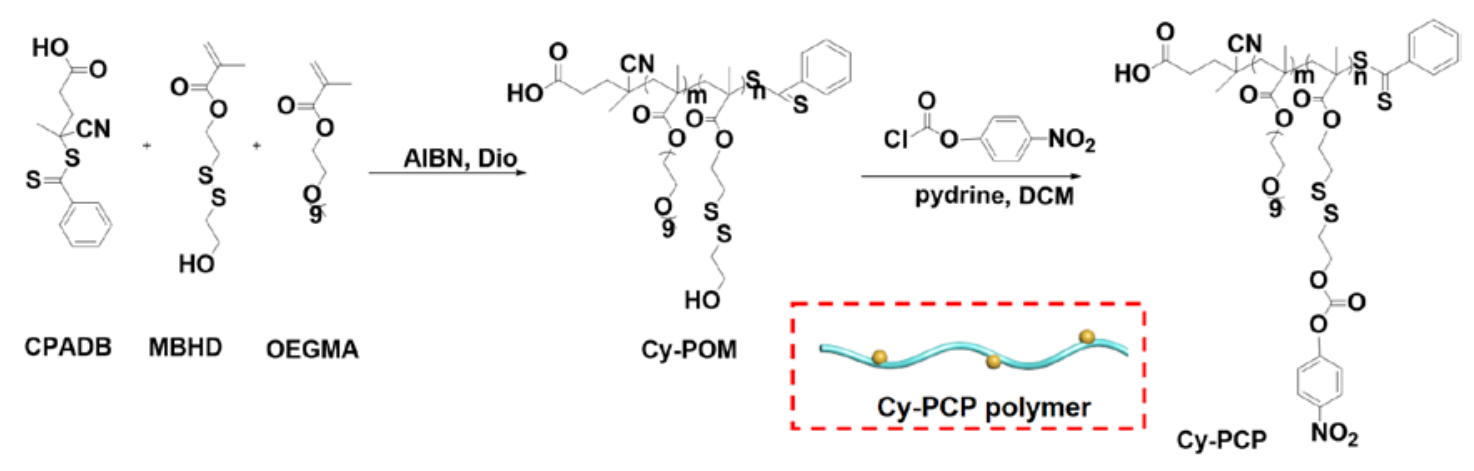

Figure S5 The synthetic route of Cy-POM and Cy-PCP polymers.
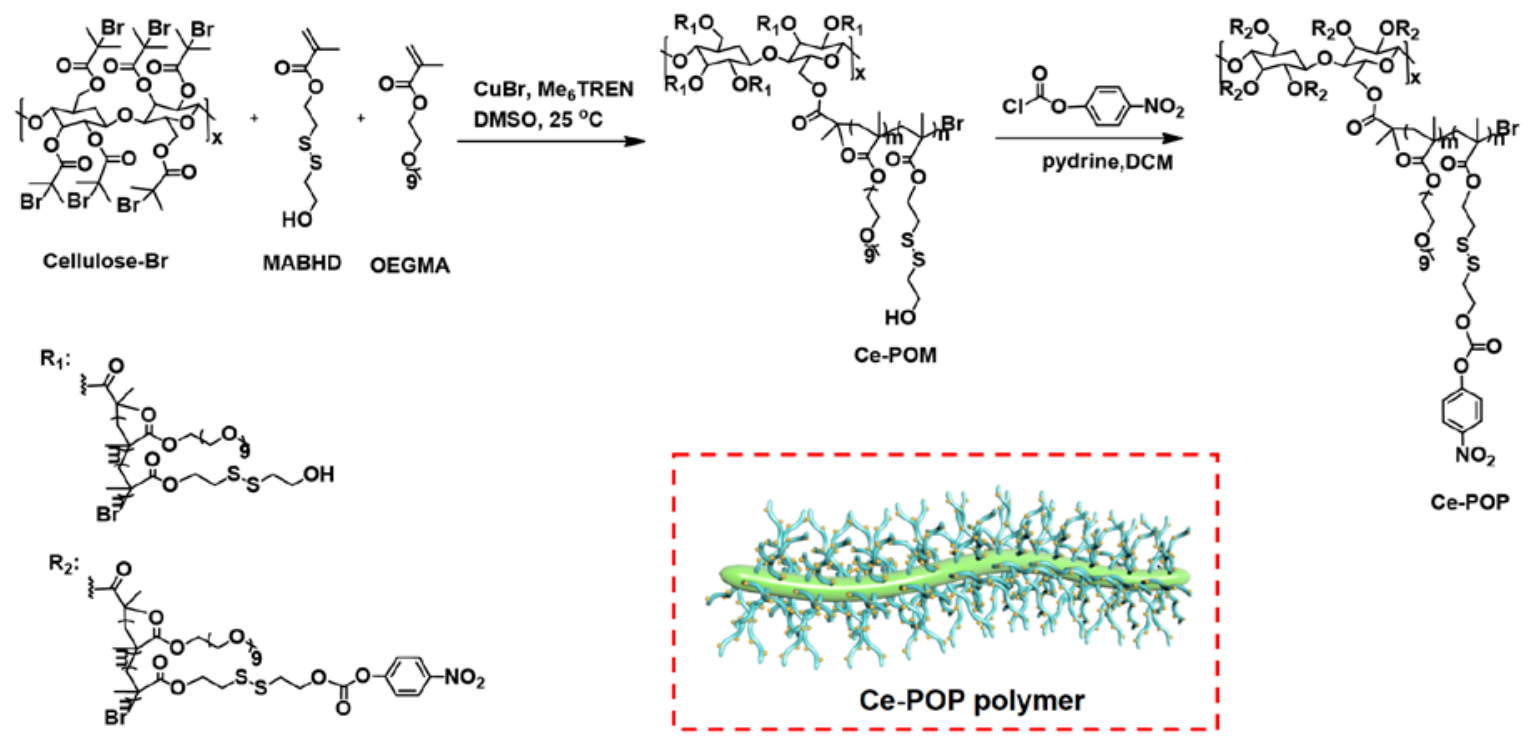

Figure S6 The synthetic route of Ce-POM and Ce-POP polymers. 


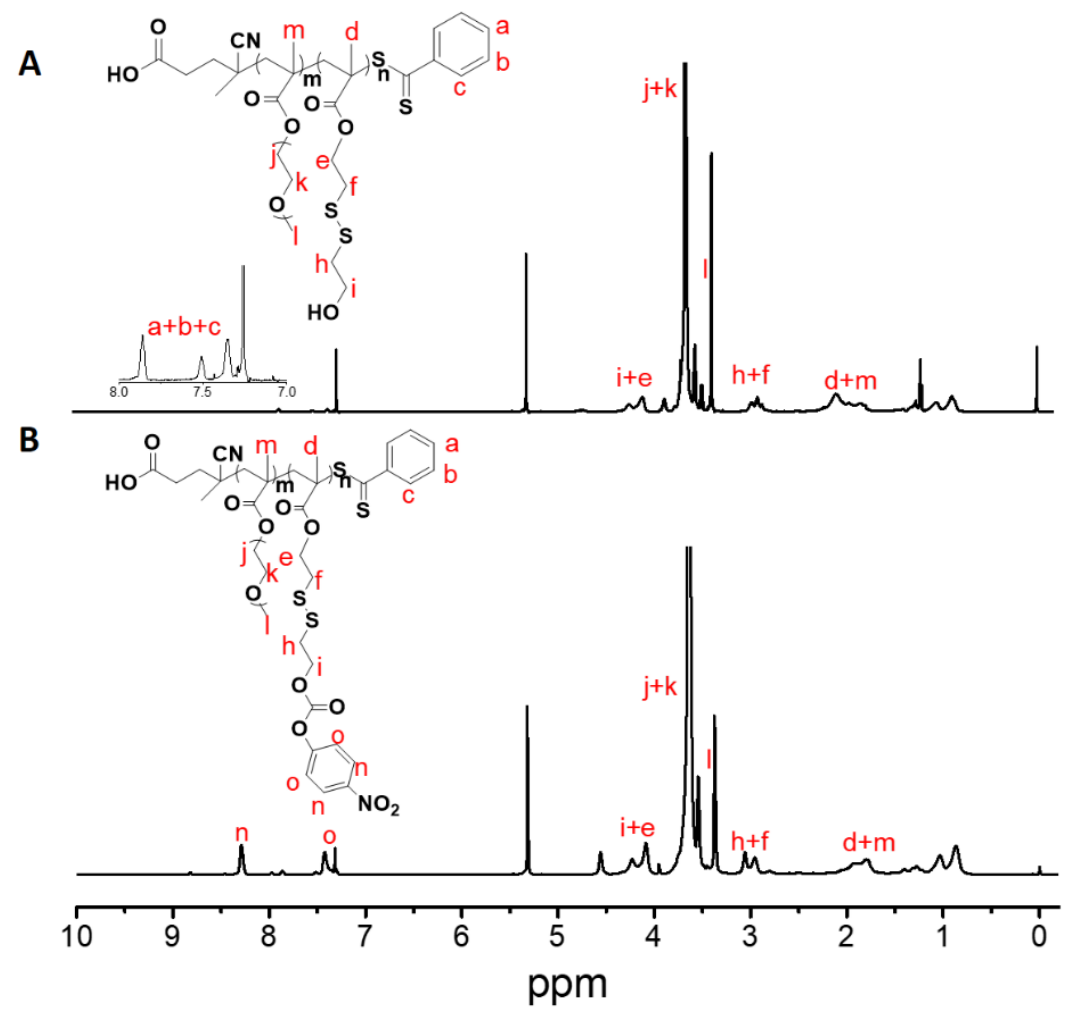

Figure S7. ${ }^{1} \mathrm{HNMR}$ spectra of Cy-POM (A) and Cy-PCP (B) detected in $\mathrm{CDCl}_{3}$. 


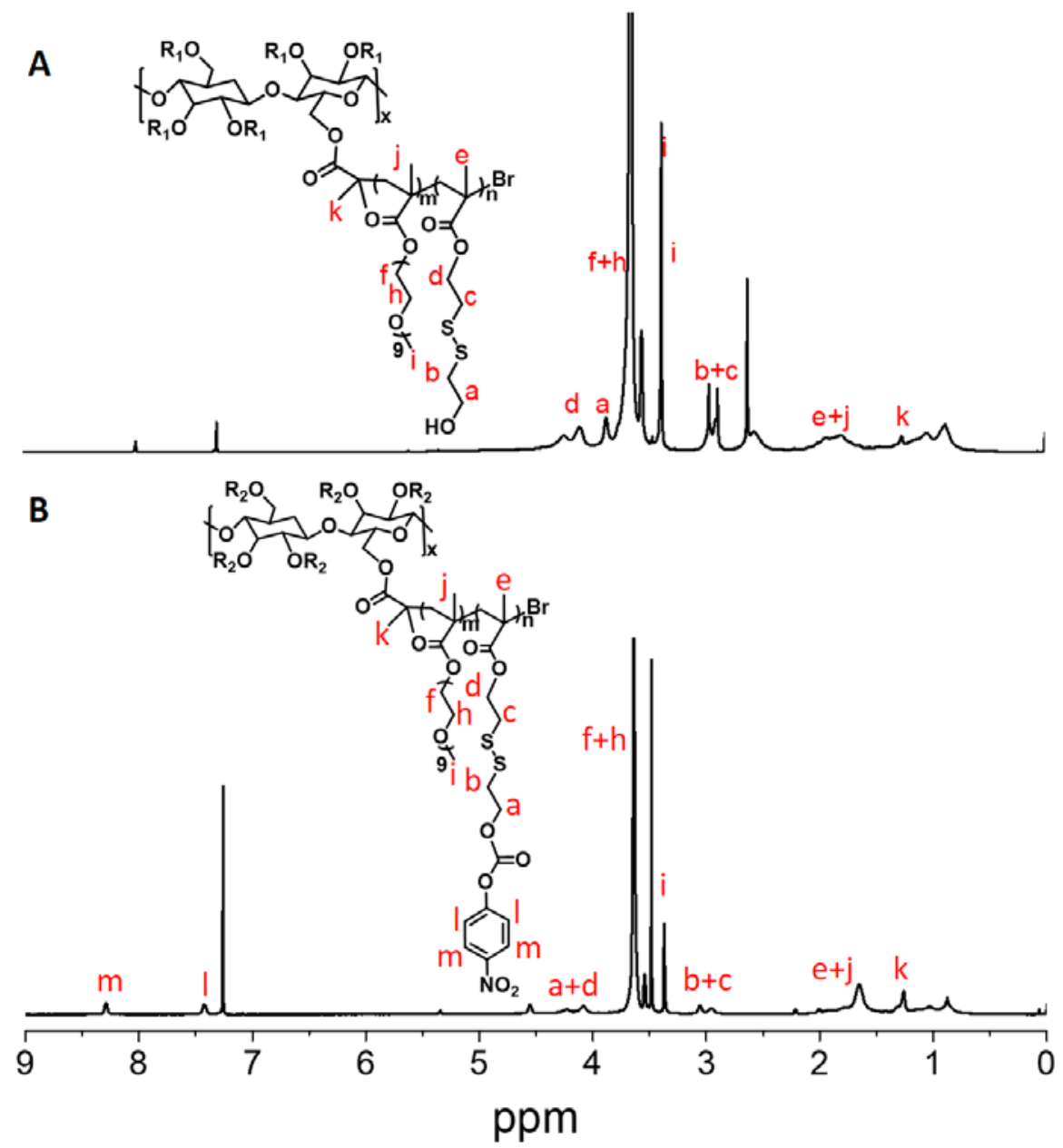

Figure S8. ${ }^{1} \mathrm{HNMR}$ spectra of Ce-POM (A) and Ce-POP (B) detected in $\mathrm{CDCl}_{3}$. 


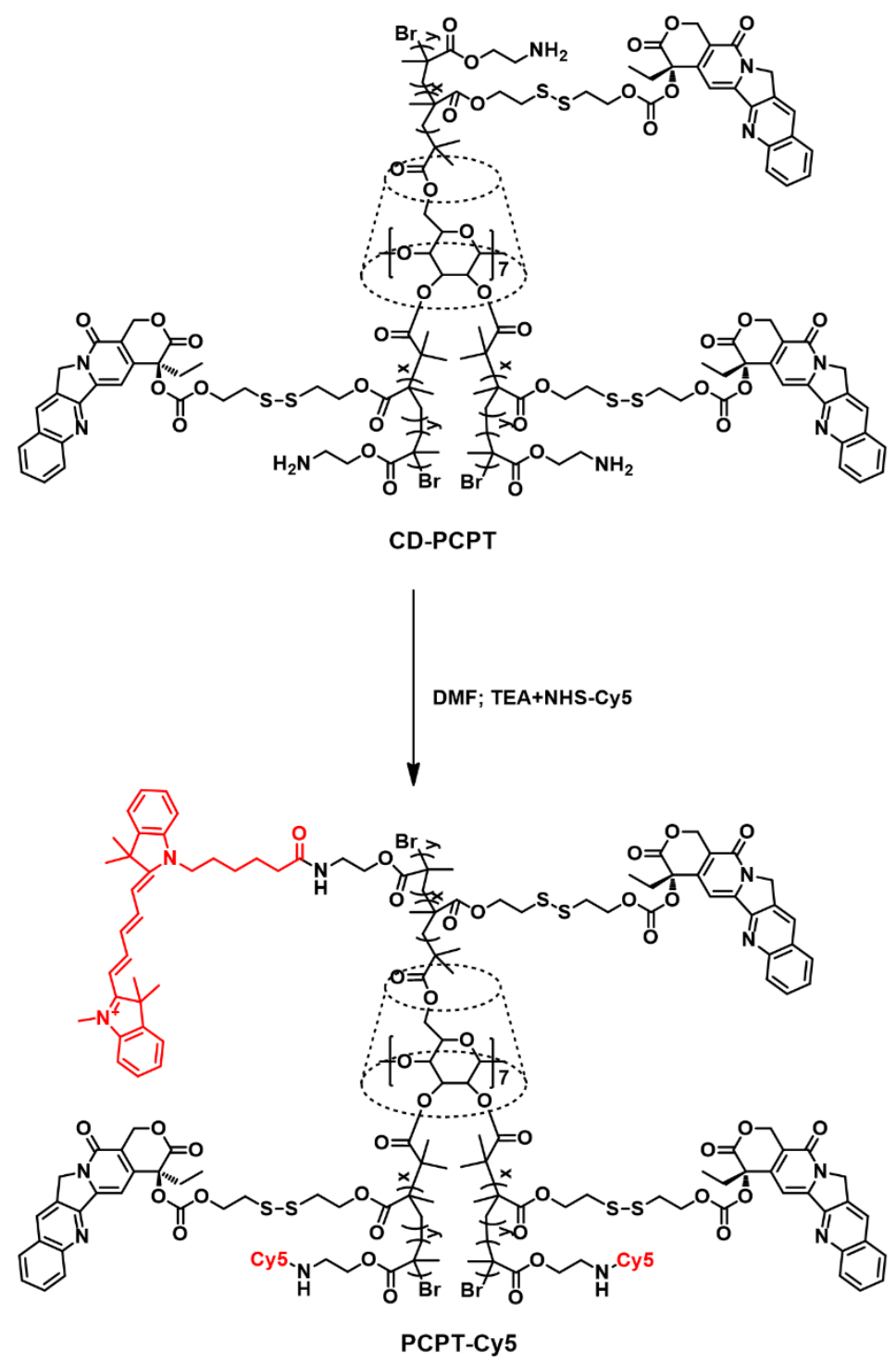

Figure S9 The synthetic route of PCPT-Cy5. 
A

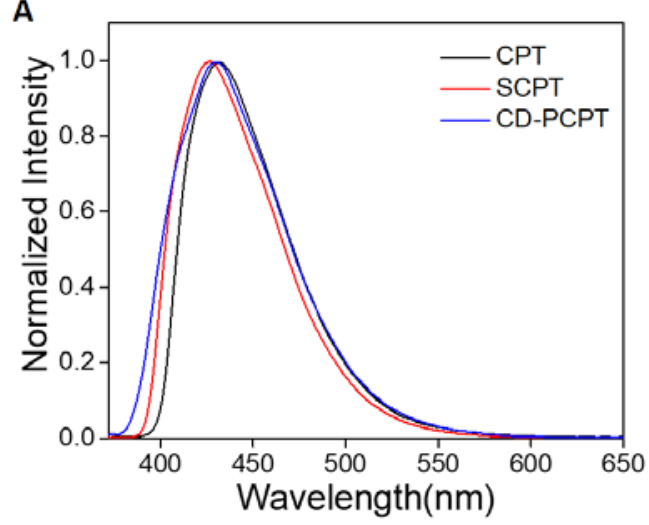

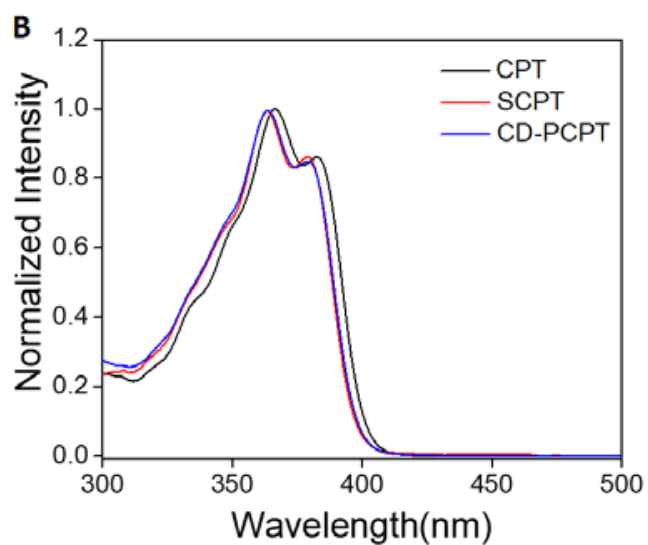

Figure S10. The fluorescent spectra (A) and UV spectra (B) of CPT, SCPT and CDPCPT in DMF media.
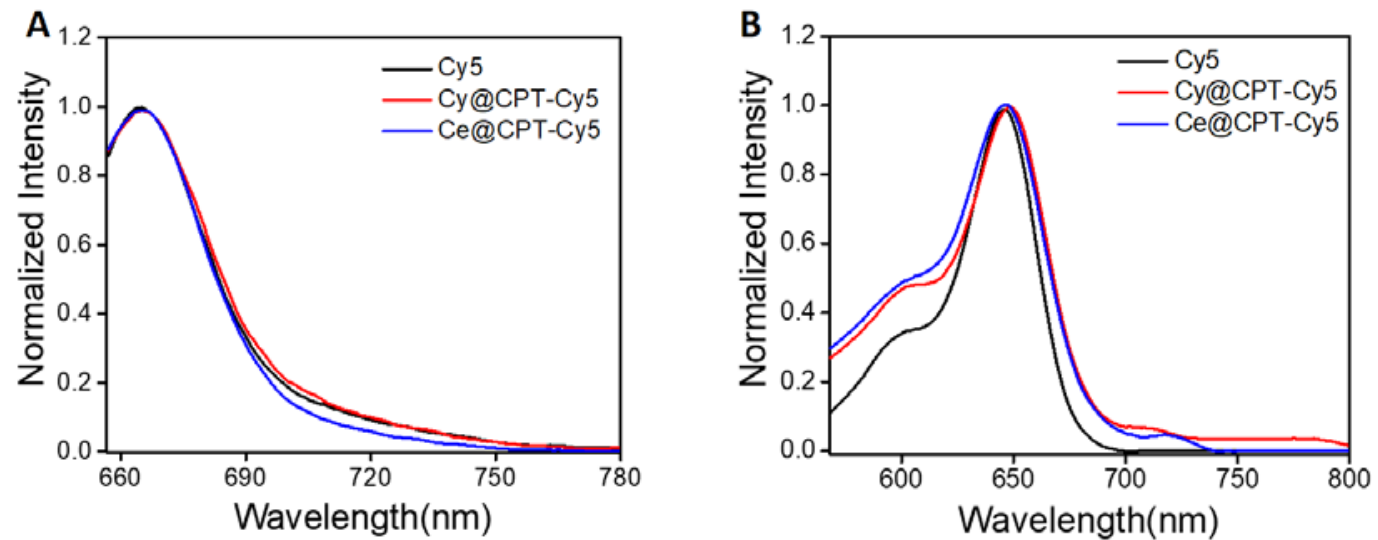

Figure S11. The UV spectra (A) and fluorescent spectra (B) of Cy5, Сy@CPT-Cy5 and Ce@CPT-Cy5 in water media. 


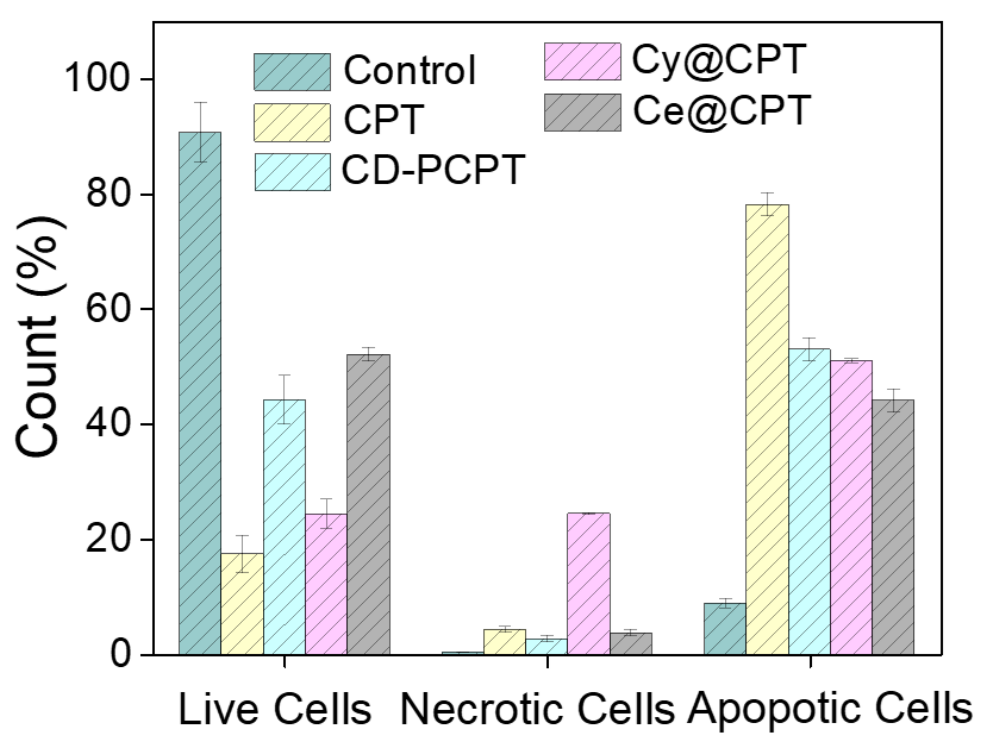

Figure S12. The statistical analysis statistics of the apoptotic and necrotic cell percentage by Flow cytometry. The data were presented as mean \pm SD ( $n=3)$.

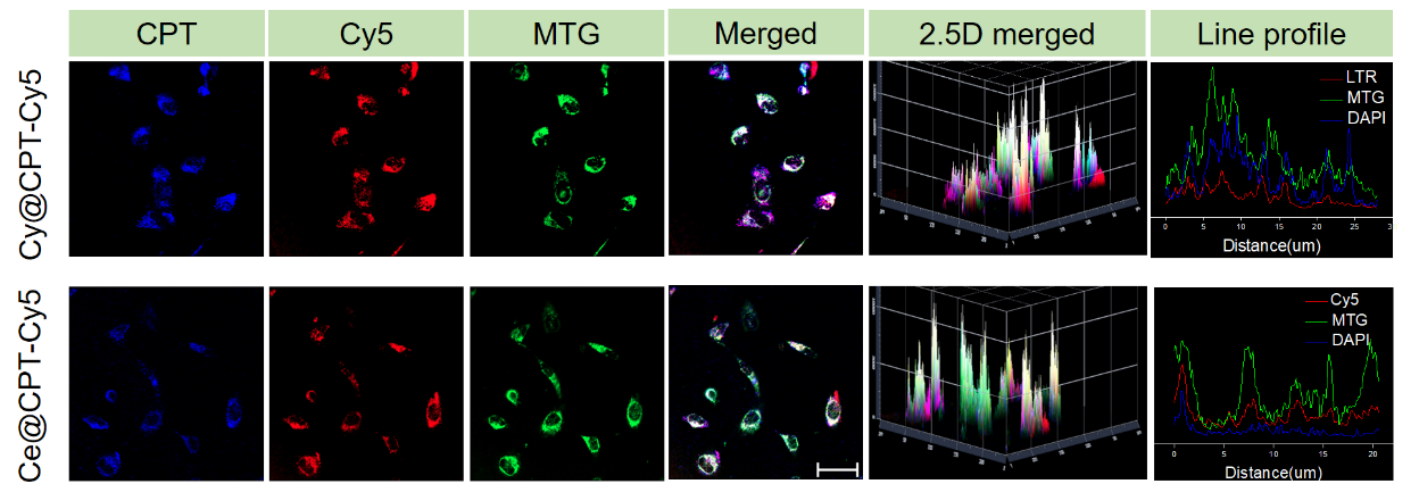

Figure S13. Cellular uptake evaluation by CLSM images, after incubated HeLa cells with Cy@CPT-Cy5 or Ce@CPT-Cy5 micelles for 6h, the cells were stained with MitoTracker Green FM (MTG). The fluorescence of CPT, Cy5 and MTG were labeled blue, red and green. Scale bars: 50um. 

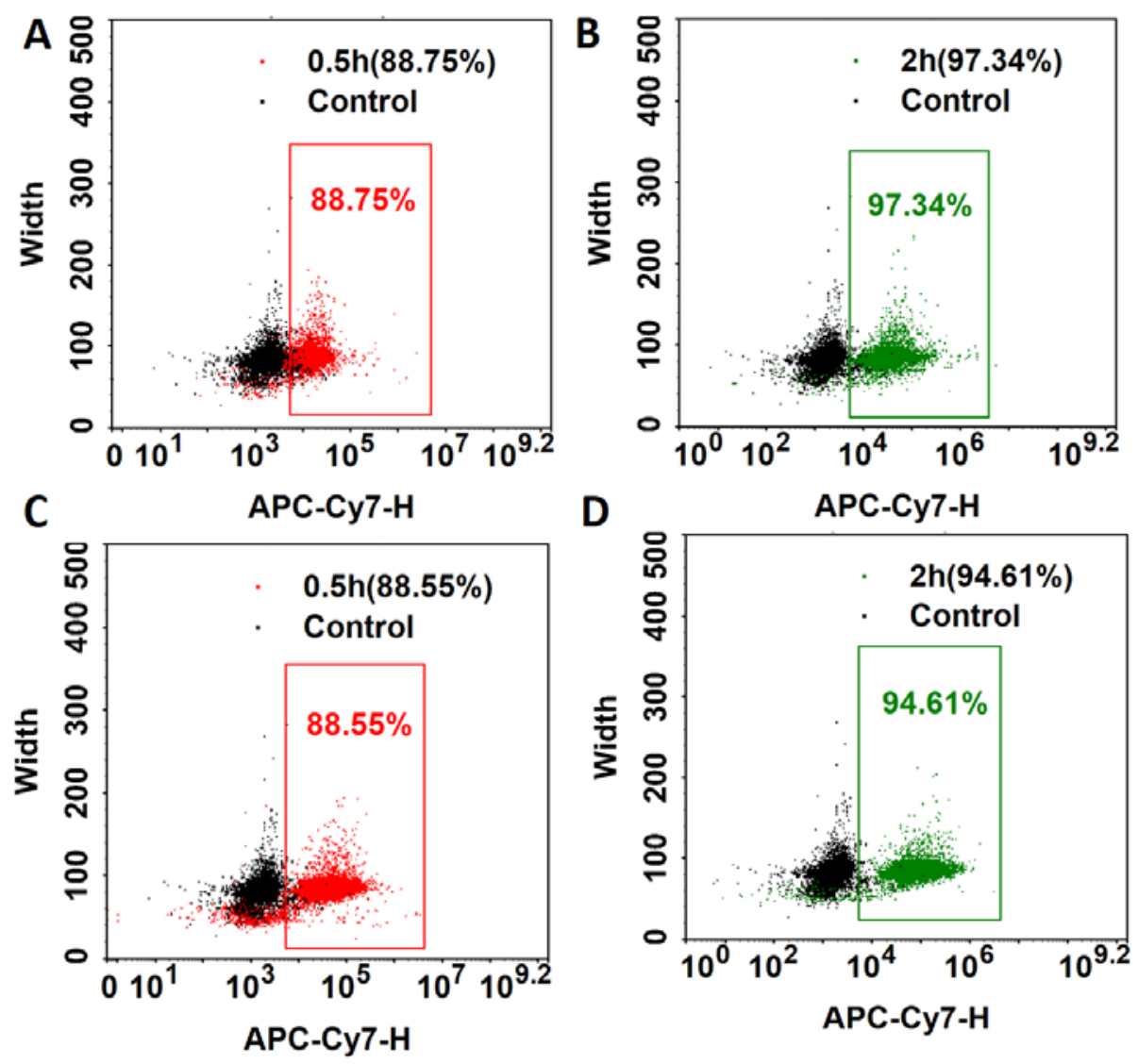

Figure S14. The flow cytometry analysis of Hela cells treated with Cy@CPT-Cy5 (A and B) and Ce@CPT-Cy5 micelles (C and D) for 0.5h and 2h. 


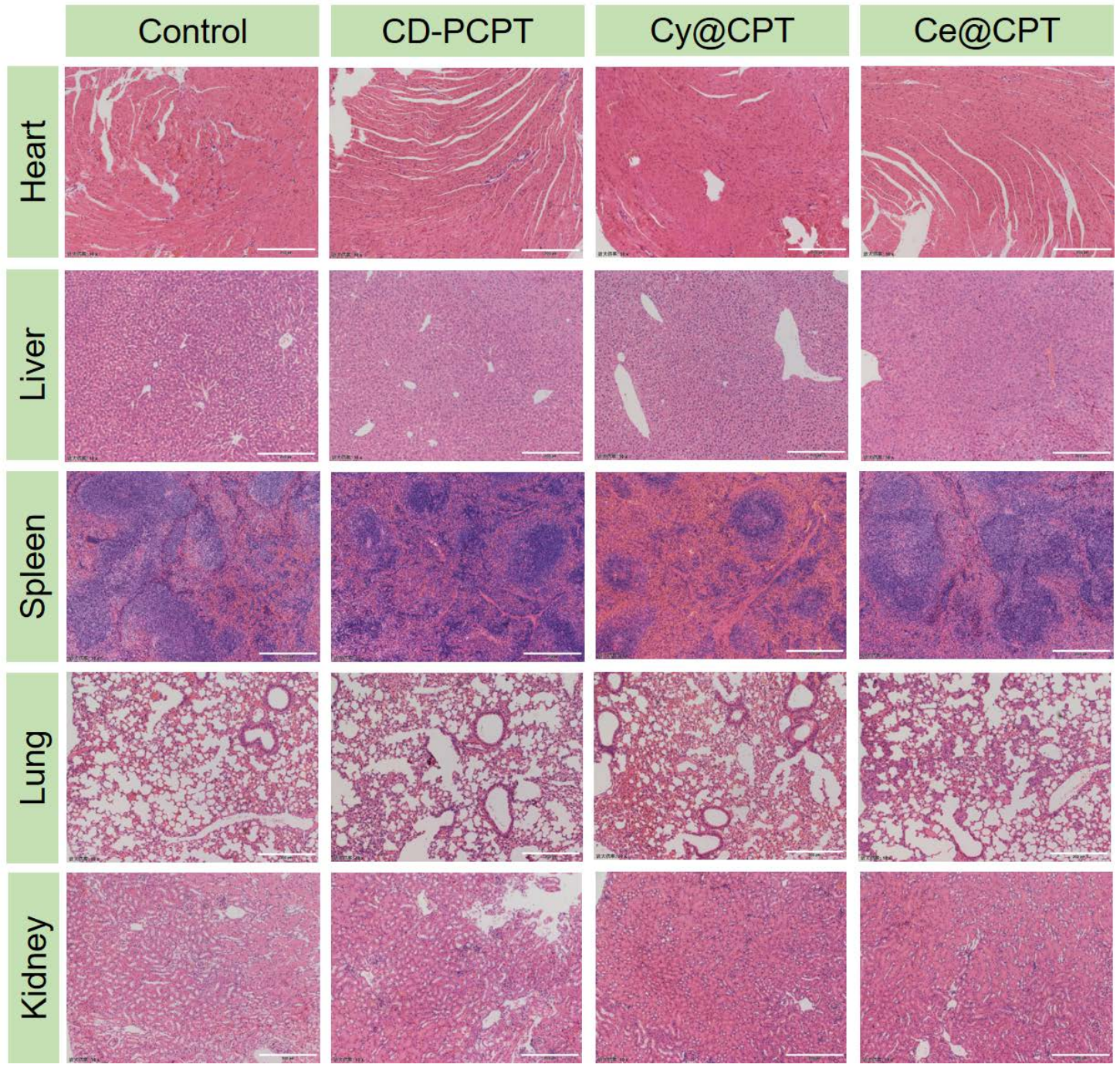

Figure S15 H\&E staining images of major organs (Heart, Liver, Spleen, Lung and Kidney) from PBS (control), CD-PCPT, Cy@CPT and Ce@CPT administrated mice. Scale bars: $200 \mu \mathrm{m}$. 
Table S1. GPC analysis of the polymers. $M_{\mathrm{n}, \mathrm{GPC}}$ is number-average molecular weight, $M_{\mathrm{w}, \mathrm{GPC}}$ is weight-average molecular weight, $M_{W} / M_{n}$ is polydispersity index (PDI).

\begin{tabular}{lccc}
\hline Polymer & $\begin{array}{c}M_{\mathrm{n}, \mathrm{GPC}} \\
\left(\mathrm{g} \mathrm{mol}^{-1}\right)\end{array}$ & $M_{\mathrm{w}, G P C}$ & $\begin{array}{c}M_{W} / M_{n} \\
(\mathrm{~nm})\end{array}$ \\
\hline Cy-POM & 4600 & 5400 & 1.16 \\
Cy-PCP & 5200 & 6000 & 1.15 \\
Ce-Br & 19000 & 29000 & 1.53 \\
Ce-POP & 29000 & 52000 & 1.77 \\
\hline
\end{tabular}

